\section{Inflammatory arthritis due to immune checkpoint inhibitors: challenges in diagnosis and treatment}

\author{
"Using immunomodulatory therapies to treat inflammatory \\ arthritis is not without associated risk, but should be considered \\ early in a patient's course to preserve quality of life and \\ prevent structural damage."
}

First draft submitted: 3 October 2016; Accepted for publication: 18 November 2016; Published online: 21 December 2016

Keywords: cancer $\bullet$ inflammatory arthritis $\bullet$ immune checkpoint inhibitors $\bullet$ immunotherapy

Immune checkpoint inhibitors (ICIs) are expanding treatment options and increasing survival for patients in selected advanced malignancies [1-7]. Ipilimumab, targeting CTLA-4, pembrolizumab and nivolumab, targeting PD-1, and atezolizumab, targeting PD-L1, have been US FDA-approved for a variety of indications including metastatic melanoma $[2,4,8,9]$, non-small-cell lung cancer [7,10], renal cell carcinoma [5], Hodgkin's lymphoma [11], head and neck cancers [12] and urothelial carcinoma [13]. Multiple agents targeting the PD-1/PD-L1 pathway and other immune checkpoint molecules are also in clinical development. The adverse events secondary to these therapies are likely related to their mechanism of action, that is, activation of T-cells and downstream inflammatory consequences. Autoantibodies have not been widely seen in immune-related adverse events (IRAEs), though hypophysitis has been shown to occur with specific autoantibody formation targeting the pituitary [14]. IRAEs may affect a variety of organs, and may vary considerably in clinical presentation and severity. Recognition of the spectrum of IRAEs is increasing, but for rheumatic adverse events, specifically inflammatory arthritis (IA), the prevalence and risk factors remain unclear. As of now, there are only case series and reports [15-18] describing clinical features and treatment of ICI-induced IA. Many clinical trials evaluat- ing ICIs did not report IA specifically as an adverse event. The challenges in diagnosing and treating IA due to immune checkpoint inhibition can be addressed through increased awareness among providers and collaboration between oncologists and rheumatologists.

\section{Diagnosis}

Awareness of IA as a potential IRAE is paramount in making the diagnosis. If patients are not asked specifically about symptoms of IA by their providers, IA may not be identified, or there may be a delay in diagnosis. Delay in diagnosis and treatment may have long-term consequences in terms of disability and joint deformities as discussed below. A similar example from rheumatology is that of diagnosing psoriatic arthritis in people with known psoriasis. Psoriatic arthritis occurs in about a third of patients with psoriasis [19] and can have diverse clinical manifestations. Increased awareness among the dermatology community, aided by the development of screening tools for psoriatic arthritis [20], has increased referral to rheumatology and thus earlier diagnosis and treatment. A similar approach could facilitate recognition of ICIinduced IA; for example, the development of a short screening tool for use by oncologists and patients receiving ICIs.

Oncology providers have limited available guidance on how to identify IA versus

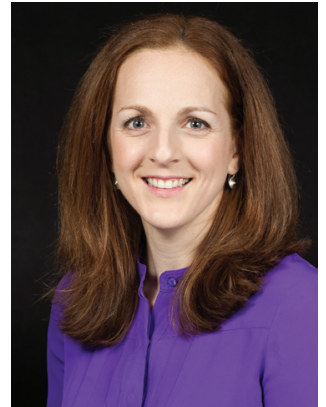

Laura C Cappelli $i^{*}, 1$ Jarushka Naidoo², Clifton O Bingham III \& Ami A Shah ${ }^{1}$

'Division of Rheumatology, Department of Medicine, Johns Hopkins School of Medicine, 5200 Eastern Avenue, Baltimore MD, 21224, USA 2Department of Oncology, Johns Hopkins School of Medicine,440 Eastern Avenue, Baltimore MD, 21224, USA *Author for correspondence: Icappel1@jhmi.edu 
other causes of joint pain. Peripheral joint pain, back and neck pain are all common problems and can have many noninflammatory causes. Providers should follow reports of new joint pain with questions to determine whether an IA may be present. If a symptom is new since receiving the ICI, this may be quite different from the presence of a symptom that was present before. Stiffness that is worse in the morning, lasting for more than $30 \mathrm{~min}$ to $1 \mathrm{~h}$, or after being sedentary may be indicative of IA. Joint swelling, warmth or erythema are also clues to an inflammatory process. Improvement of symptoms with NSAIDs or corticosteroids, but not with opiates or other pain medications, may also be suggestive. If any of these features are present or the provider is unsure, referral to rheumatology for evaluation and treatment is appropriate.

Early recognition of IA is critical, so that patients can be evaluated and treated quickly. Once a patient comes to a rheumatologist for IA of any type, rapid control of inflammation is the goal. We know from classic forms of IA, such as rheumatoid arthritis (RA), that erosive joint damage can occur early. More sensitive imaging modalities, namely MRI, have demonstrated erosions after only months of symptoms in RA [21,22]. Early treatment with immunomodulatory therapy can prevent this permanent damage. This is especially relevant as patients are responding to ICIs and living longer and as ICIs are being investigated in early-stage disease. Thus, preventing disability and preserving quality of life becomes more important in patients with a longer life expectancy.

\section{Treatment}

For other IRAEs, corticosteroids are the mainstay of treatment. For severe symptoms (grade 3 or higher by Common Terminology for Adverse Events grading), ICIs may also be held or discontinued. The duration of corticosteroid therapy is usually limited, lasting for about 4-6 weeks, and many IRAEs resolve within weeks to months of treatment. In a small subset of patients, other immunosuppressive agents are needed. The most experience using medications other than corticosteroids is in immune-related colitis, where short courses of the TNF-inhibitor, infliximab, have been used [23]. An important difference between other IRAEs and IA, relevant to treatment, appears to be the time course. Most IRAEs develop within 12-14 weeks of starting therapy and resolve with treatment within months [24]. In the limited published experience, arthritis secondary to ICIs can persist over a year after ICIs are stopped [18]. Thus, the need for continued immunosuppression may be more relevant to IA than events such as immune-related colitis, which tend to be self-limiting after management with corticosteroids or limited doses of infliximab [25]. This observation combined with potential earlier use of ICIs and better survival will necessitate a longer time than patients require during immunosuppression.

Corticosteroids can be used as part of initial therapy in IA, but due to likely prolonged treatment requirements, physicians should consider starting steroidsparing agents earlier than one would with other IRAEs. A wide variety of agents are available to treat classic forms of IA, such as RA. In RA, therapies are traditionally divided into two categories: conventional synthetic disease modifying anti-rheumatic drugs (DMARDs) and biologic DMARDs. The first category includes methotrexate, leflunomide and sulfasalazine and the latter consists of TNF-inhibitors, abatacept (T-cell costimulation blockade), tocilizumab (monoclonal antibody to IL-6 receptor), rituximab (CD-20 monoclonal antibody) and tofacitinib (Janus kinase inhibitor, the only oral biologic medication). Looking to other classic forms of IA, secukinumab (monoclonal antibody to IL-17) is approved in psoriatic arthritis and ankylosing spondylitis, with ustekinumab (monoclonal antibody to IL-12/23) approved for psoriatic arthritis. All of these agents differ in their mechanisms of action, time to efficacy, side effect profiles and potential effects on tumor response to ICIs. The oncologist and rheumatologist must balance the potential benefits of these agents to improve symptoms and prevent permanent joint damage with the potential risks, both in side effects and in effects on cancer progression. We highlight these issues by examining several drugs used in the treatment of RA and providing examples of concerns that may occur in each of these areas.

\section{Time course/likelihood of efficacy}

Thus far, there are reports of using corticosteroids, methotrexate and various TNF inhibitors successfully for treatment of ICI-induced IA $[17,18]$. Corticosteroids work quickly, within days, but methotrexate can take weeks (8-12) to reach peak effect and may not be the best choice in isolation for patients with severe symptoms. TNF inhibitors can reach peak efficacy more quickly than methotrexate, sometimes after a single dose. Some conventional synthetic DMARDs such as sulfasalazine have few concerns in terms of impairing cancer response to therapy, but are slow in action and less likely to be effective in severe disease, making them less useful.

\section{Potentially overlapping side effects}

Since patients can have more than one IRAE, there are concerns that side effects from treatments for IA may exacerbate other types of IRAEs. Sulfasalazine can 
cause a severe allergic reaction with rash and may not be the best choice in patients experiencing rash from ICIs. Tocilizumab and tofacitinib have been associated with bowel perforation in patients with diverticulitis, so may not be a good choice in those with prior colitis. On the other hand, picking treatments such as TNF inhibitors that also have efficacy in colitis may be preferable in patients who have already experienced that type of IRAE.

\section{Effects on tumor progression}

TNF inhibitors have been used for other IRAEs for short courses, but have not been used for an extended period of time as in IA. When examining classic forms of IA, a large amount of data exists regarding associated risks of TNF inhibitor use over time. Although there are concerns about increased risk of de novo solid tumor development in a trial of etanercept for vasculitis [26], there are mounting data from large observational studies that TNF-inhibitors do not substantially increase risk for de novo cancers in patients with RA, including recently published studies focusing on invasive melanoma and lymphoma [27,28]. Though these data are reassuring for patients without a history of cancer, it is not directly applicable to patients treated with ICIs who already have a known malignancy. Since the exact mechanisms by which ICIs achieve their antitumor effects are unknown, only theoretical risks can be assessed. Another drug used to treat RA, abatacept, is a soluble CTLA-4:IgG Fc construct which blocks T-cell costimulation, and may thus impair the antitumor effect of ICIs. As more is understood about the mechanisms through which ICIs exert an antitumor effect, it may be possible to rationalize the choice of immunosuppressive

\section{References}

1 Robert C, Schachter J, Long GV et al. Pembrolizumab versus ipilimumab in advanced melanoma. N. Engl. J. Med. 372(26), 2521-2532 (2015).

2 Robert C, Thomas L, Bondarenko I et al. Ipilimumab plus dacarbazine for previously untreated metastatic melanoma. N. Engl. J. Med. 364(26), 2517-2526 (2011).

3 Weber JS, D’Angelo SP, Minor D et al. Nivolumab versus chemotherapy in patients with advanced melanoma who progressed after anti-CTLA-4 treatment (CheckMate 037): a randomised, controlled, open-label, Phase 3 trial. Lancet Oncol. 16(4), 375-384 (2015).

4 Larkin J, Chiarion-Sileni V, Gonzalez R et al. Combined nivolumab and ipilimumab or monotherapy in untreated melanoma. N. Engl. J. Med. 373(1), 23-34 (2015).

5 Motzer RJ, Escudier B, McDermott DF et al. Nivolumab versus everolimus in advanced renal-cell carcinoma. N. Engl. J. Med. 373(19), 1803-1813 (2015).

6 Borghaei H, Paz-Ares L, Horn L et al. Nivolumab versus agent, so as not to obviate the mode of action of the relevant ICI. This is an important area for future research.

\section{Summary}

Challenges in diagnosing and treating IA secondary to ICIs can be overcome with a collaborative approach between rheumatology and oncology. The development of screening tools, as well as evaluation and treatment of algorithms for IA, will aid in the management of these patients. Using immunomodulatory therapies to treat IA is not without associated risk, but should be considered early in a patient's course to preserve quality of life and prevent structural damage.

\section{Financial \& competing interests disclosure}

$\mathrm{CO}$ Bingham has served as a consultant for Bristol-Myers Squibb. J Naidoo has served as a consultant for Bristol-Myers Squibb and has received honoraria from Bristol-Myers Squibb and AstraZeneca. This study was supported by the Jerome L Greene Foundation Scholar Award and the National Institute of Arthritis and Musculoskeletal and Skin Diseases (P30AR053503; K23-AR061439). The authors have no other relevant affiliations or financial involvement with any organization or entity with a financial interest in or financial conflict with the subject matter or materials discussed in the manuscript apart from those disclosed.

No writing assistance was utilized in the production of this manuscript.

\section{Open access}

This work is licensed under the Attribution-NonCommercialNoDerivatives 4.0 Unported License. To view a copy of this license, visit http://creativecommons.org/licenses/by-nc$\mathrm{nd} / 4.0 /$

docetaxel in advanced nonsquamous non-small-cell lung cancer. N. Engl. J. Med. 373(17), 1627-1639 (2015).

7 Herbst RS, Baas P, Kim DW et al. Pembrolizumab versus docetaxel for previously treated, PD-L1-positive, advanced non-small-cell lung cancer (KEYNOTE-010): a randomised controlled trial. Lancet 387(10027), 1540-1550 (2016).

8 Robert C, Long GV, Brady B et al. Nivolumab in previously untreated melanoma without BRAF mutation. N. Engl. J. Med. 372(4), 320-330 (2015).

9 Robert C, Ribas A, Wolchok JD et al. Anti-programmeddeath-receptor-1 treatment with pembrolizumab in ipilimumab-refractory advanced melanoma: a randomised dose-comparison cohort of a Phase 1 trial. Lancet 384(9948), 1109-1117 (2014).

10 Brahmer J, Reckamp KL, Baas P et al. Nivolumab versus docetaxel in advanced squamous-cell non-small-cell lung cancer. N. Engl. J. Med. 373(2), 123-135 (2015).

11 Ansell SM, Lesokhin AM, Borrello I et al. PD-1 blockade with nivolumab in relapsed or refractory Hodgkin's lymphoma. N. Engl. J. Med. 372(4), 311-319 (2015). 
12 Seiwert TY, Burtness B, Mehra R et al. Safety and clinical activity of pembrolizumab for treatment of recurrent or metastatic squamous cell carcinoma of the head and neck (KEYNOTE-012): an open-label, multicentre, Phase 1b trial. Lancet Oncol. 17(7), 956-965 (2016).

13 Powles T, Eder JP, Fine GD et al. MPDL3280A (anti-PD-L1) treatment leads to clinical activity in metastatic bladder cancer. Nature 515(7528), 558-562 (2014).

14 Iwama S, De Remigis A, Callahan MK, Slovin SF, Wolchok JD, Caturegli P. Pituitary expression of CTLA-4 mediates hypophysitis secondary to administration of CTLA-4 blocking antibody. Sci. Transl. Med. 6(230), 230ra45 (2014).

15 Chan MM, Kefford RF, Carlino M, Clements A, Manolios $\mathrm{N}$. Arthritis and tenosynovitis associated with the antiPD1 antibody pembrolizumab in metastatic melanoma. J. Immunother. 38(1), 37-39 (2015).

16 de Velasco G, Bermas B, Choueiri TK. Autoimmune arthropathy and uveitis as complications of programmed death 1 inhibitor treatment. Arthritis Rheumatol. 68(2), 556-557 (2016).

17 Law-Ping-Man S, Martin A, Briens E, Tisseau L, Safa G. Psoriasis and psoriatic arthritis induced by nivolumab in a patient with advanced lung cancer. Rheumatology (Oxford) doi:10.1093/rheumatology/kew281 (2016) (Epub ahead of print).

18 Cappelli LC, Gutierrez AK, Baer AN et al. Inflammatory arthritis and sicca syndrome induced by nivolumab and ipilimumab. Ann. Rheum. Dis. doi:10.1136/ annrheumdis-2016-209595 (2016) (Epub ahead of print).

19 Gladman D, Antoni C, Mease P, Clegg D, Nash P. Psoriatic arthritis: epidemiology, clinical features, course, and outcome. Ann. Rheum. Dis. 64(Suppl. 2), ii14-ii17 (2005).

20 Olivieri I, D’Angelo S, Palazzi C, Padula A. Advances in the management of psoriatic arthritis. Nat. Rev. Rheumatol. 10(9), 531-542 (2014).

21 Ostergaard M, Hansen M, Stoltenberg M et al. New radiographic bone erosions in the wrists of patients with rheumatoid arthritis are detectable with magnetic resonance imaging a median of two years earlier. Arthritis Rheum. 48(8), 2128-2131 (2003).

22 Mcqueen FM, Stewart N, Crabbe J et al. Magnetic resonance imaging of the wrist in early rheumatoid arthritis reveals a high prevalence of erosions at four months after symptom onset. Ann. Rheum. Dis. 57(6), 350-356 (1998).

23 Beck KE, Blansfield JA, Tran KQ et al. Enterocolitis in patients with cancer after antibody blockade of cytotoxic T-lymphocyte-associated antigen 4. J. Clin. Oncol. 24(15), 2283-2289 (2006).

24 Weber JS, Kahler KC, Hauschild A. Management of immune-related adverse events and kinetics of response with ipilimumab. J. Clin. Oncol. 30(21), 2691-2697 (2012).

25 O'connor A, Marples M, Mulatero C, Hamlin J, Ford AC. Ipilimumab-induced colitis: experience from a tertiary referral center. Therap. Adv. Gastroenterol. 9(4), 457-462 (2016).

26 Silva F, Seo P, Schroeder DR et al. Solid malignancies among etanercept-treated patients with granulomatosis with polyangiitis (Wegener's): long-term followup of a multicenter longitudinal cohort. Arthritis Rheum. 63(8), 2495-2503 (2011).

27 Mercer LK, Askling J, Raaschou P et al. Risk of invasive melanoma in patients with rheumatoid arthritis treated with biologics: results from a collaborative project of 11 European biologic registers. Ann. Rheum. Dis. doi:10.1136/ annrheumdis-2016-209285 (2016) (Epub ahead of print).

28 Mercer LK, Galloway JB, Lunt M et al. Risk of lymphoma in patients exposed to antitumour necrosis factor therapy: results from the British Society for Rheumatology Biologics Register for Rheumatoid Arthritis. Ann. Rheum. Dis. doi:10.1136/annrheumdis-2016-209389 (2016)(Epub ahead of print). 